\title{
Tolerance Specification Optimization for Economic and Ecological Sustainability
}

\author{
Steven Hoffenson, Andreas Dagman, Rikard Söderberg \\ Department of Product and Production Development, Chalmers University of Technology, \\ Gothenburg, Sweden \\ \{stevenh, andreas.dagman, rikard.soderberg\} @chalmers.se
}

\begin{abstract}
In the final stages of product development, dimensional tolerances are specified by designers to ensure high functionality at low costs. A traditional approach to this decision-making process is to minimize economic losses to the manufacturer and the consumer. This paper presents a new approach for tolerance allocation optimization that considers sustainability not only from economic costs but also ecological costs. The framework is formulated as a multiobjective optimization problem and explored with a case study on the design of an automotive body panel. Results of the case study include Pareto frontiers of non-dominated optimal solutions along with a parametric study to explore the influence of material choice on the results.
\end{abstract}

Keywords: tolerance allocation, variation propagation, sustainability, cost minimization, multi-objective optimization

\section{Introduction}

The quality of products is partially determined when designers select tolerances to specify in engineering drawings. Tolerance selection is a necessary step in product design since no product dimension can be manufactured with perfect accuracy, and a tradeoff exists because tighter tolerances increase manufacturing costs and looser tolerances often inhibit the assemblability and functionality of the product. Poor assemblability can result in a high number of defective parts and products being discarded as well as time wasted. Poor functionality, which is typically associated with consumer perception of quality, can result in products being discarded and replaced early in the expected life cycle along with customer dissatisfaction and lowered brand reputation. Discarding parts and products is not only an economic concern for manufacturers and consumers, but it is also an ecological issue, since resource and energy usage in the production and disposal of replacement parts can be significant.

This article presents a multi-objective modeling and optimization framework for considering the economic and ecological ramifications of tolerance selection in the final stages of product design. The design approach is demonstrated through a case study that considers the tolerance specifications for an automotive body panel, where 
specified tolerances influence a critical dimension that affects the product's assembled functionality. Results include Pareto frontiers as well as a parametric study to introduce the influence of material choice on the optimization results.

The ensuing section discusses previous literature on tolerance analysis and variation propagation, previous efforts in tolerance-cost minimization, and techniques for environmental impact assessment. Section 3 presents the framework, methodology, and models used in the case study. Optimization results for the tolerances in the automotive panel are delivered in Section 4 and discussed in Section 5. The final section offers conclusions and discusses the broader implications of the work.

\section{Background}

Dimensional tolerance allocation is a subject currently addressed at several conferences and journals, and there is abundant literature discussing years of investigation on variation propagation and cost optimization in the domain of tolerance specification. Sections 2.1 and 2.2 discuss the state of the art in these two areas, respectively. The novelty of the present approach is the addition of ecological sustainability as an objective for tolerance allocation, and Section 2.3 provides a brief background of research on ecological sustainability and how it is managed in general applications.

\subsection{Tolerance Allocation and Variation Propagation}

Tolerances of product dimensions must be chosen carefully due to the aforementioned tradeoff between quality and cost, and they are often chosen based on the ways that each dimension influences the functional requirements of the part or product. Variance in one measurement of a part or product is said to propagate and influence the resulting variance in other measurements. Techniques to calculate the relationships and propagation between specified tolerances and critical dimension variations are often referred to as tolerance analysis and synthesis [1].

Designers typically set tolerances based on either worst-case design scenarios or statistical analyses of tolerance probability distributions and the associated critical dimension variations. This is most simply calculated using linear or linearized tolerance accumulation models, where the tolerances of components assembled in series are summed or root-sum-squared to predict the variation across the length of the whole [2]. A more common method is statistical tolerancing, where researchers and practitioners typically assume normal distributions for prescribed tolerances and calculate the distribution of the critical dimension [3]. When this calculation is impractical due to complex geometries, Monte Carlo methods are employed, in which a number of randomly-selected tolerances are generated as inputs to measure the resulting output variations [4].

All of these methods of analyzing and synthesizing tolerances have been implemented in a variety of commercial and proprietary computer software packages to aid in tolerance allocation and, in some cases, optimization $[5,6]$. 


\subsection{Tolerance-Cost Minimization}

Typical tolerance optimization is conducted with the objective of minimizing manufacturing costs, seeking to improve the manufacturer's economic sustainability while preserving product functionality. The two challenges in doing so are in understanding the relationships between specified tolerances and critical dimension variations, as discussed in the previous section, and in modeling the relationships between specified tolerances and costs. Data linking manufacturing costs and tolerances depend on a number of environmental factors and are typically proprietary, so researchers often use simple mathematical functions to describe these relationships for various manufacturing processes [7]. In many cases, the production of a component has more than one eligible manufacturing process, and piecewise functions or discrete tolerance-cost points can then be used for optimization [8,9]. Curves are generally fit to a set of costtolerance data when available, but it is common for researchers to present methods using assumed or generic cost-tolerance curves due to either an absence of data or unwillingness to publish proprietary data. One popular function is the reciprocal function, shown in Equation (1) where $c$ is cost, $t$ is tolerance, and a and $\mathrm{b}$ are parameters fit to match actual or estimated costs [10-12].

$$
c=\mathrm{a}+\mathrm{b} / t
$$

Some previous work in this area treats costs as losses to the manufacturer, where the loss is the difference between the cost to manufacture a certain tolerance and the minimum possible manufacturing cost, which is typically associated with loose tolerances [13-15]. This removes fixed costs from the equation and allows comparison of financial costs with functionality losses due to loose tolerances as described by Taguchi et. al [16]. The process capability index is commonly used in this work to measure process performance, normalized to three standard deviations from the mean of a tolerance distribution $[17,18]$. Söderberg [13] extends the loss function technique with an additional objective representing loss to the customer, as parts with looser tolerances are more likely to fail early during the use phase of a product.

\subsection{Ecological Sustainability Metrics and Assessment Tools}

In addition to influencing manufacturing costs, product quality for the manufacturer, and quality to the customer, tolerance selection affects the ecological impact of a product. Factors including choices of manufacturing processes, time and electricity requirements, material usage, and rates of defective parts produced link tolerances with ecological sustainability.

Ecological, or environmental, sustainability has been increasingly studied and debated in recent decades, particularly in the context of Life Cycle Assessment (LCA) or Life Cycle Engineering (LCE) techniques, which specifically target the cradle-tograve impact of products and processes [19]. A number of environmental impact databases, standards, and software packages have been proposed to aid in designing for the health of the planet. These measurement tools include extensive environmental impact databases that associate ecological impacts with various human actions, and 
many of them include user interfaces to aid in identifying inputs and analyzing outputs $[20,21]$. Since the outputs typically fall under different categories of impact, such as resource depletion, greenhouse gas emissions, air pollution, water pollution, and landfill use, the tools use different techniques for presenting the results in a manageable format. These include equating ecological impacts with monetary values [21] or normalizing them against an average consumer's annual impact [22].

\section{$3 \quad$ Methodology}

The present study combines tools and techniques from the literature to present a framework for analysis and optimization of tolerances to minimize costs and ecological impacts. The approach is illustrated in Figure 1.

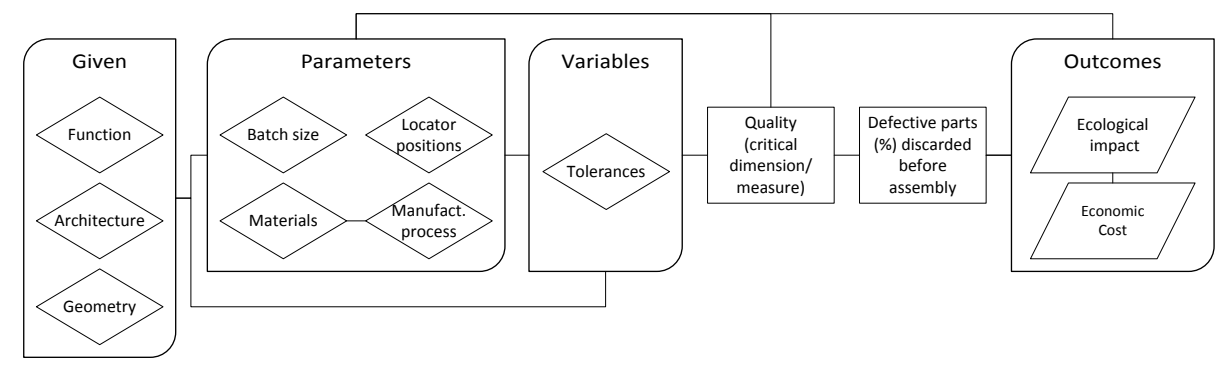

Fig. 1. Framework of product tolerance-cost-environmental-impact relationships

Here, the late-stage design problem is considered where the product function, architecture, and geometry have been decided upon, and batch size, locator positions, materials and manufacturing processes are parameters. Parameters are not allowed to vary during optimization, but they may be modified between optimizations to demonstrate how they influence the solution; this is referred to as a parametric study, and Section 4.2 shows the results of such a study on material choice. The ensuing subsections describe models that link the input parameters and variables to the outcomes in Figure 1, and with them a bi-objective optimization problem is formulated.

\subsection{Modeling}

The following sub-sections describe the models used to calculate the links in Figure 1 between tolerance specifications and quality, manufacturing parameters and cost, and manufacturing parameters and environmental impact. The models are built around the case study of a D-pillar in an automotive vehicle body, which is comprised of two stamped sheet metal components.

Variation Propagation. To model the propagation of variation from prescribed tolerances to critical functional dimensions, the software package RD\&T is used [13]. This program is designed specifically for the purpose of analyzing variation propaga- 
tion in complex geometries and includes a graphical user interface for creating models and visualizing results. The D-pillar used in this paper is shown in Figure 2, and it is subject to manufacturing variations in places where the parts are supported by other frame components and at the mating surface of the two parts. The functional requirement is that the three-dimensional position of the upper-rear corner, denoted with black squares in Figure 2, be located near the nominally designed coordinates.

The allowed variation for these critical dimensions is unclear and depends to some extent on the judgment of the designer and the design of the connecting parts. In this case, the variation in all three coordinate dimensions is allowed within one millimeter, but parametric studies are recommended to understand the sensitivity of the results to this decision.

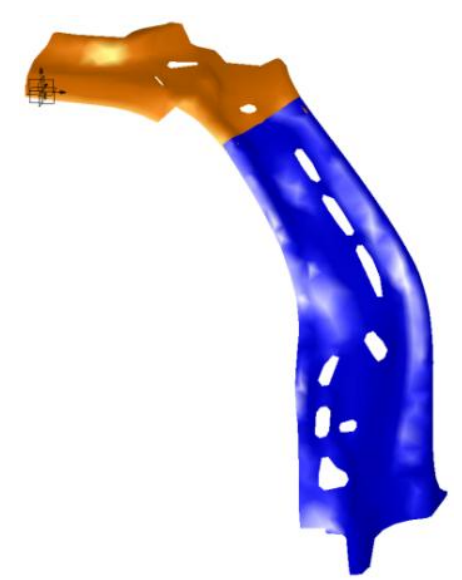

Fig. 2. D-pillar model in RD\&T

Cost Modeling. As previously discussed, cost data on manufacturing processes are not widely available or publishable. In this study, like in much of the literature, a reciprocal manufacturing cost function is assumed with the form of Equation (1). As in Ding, the equation parameters are set at $\mathrm{a}=0, \mathrm{~b}=1[11]$.

Ecological Impact Modeling. Since the objective of this article is to discuss the tradeoffs among costs and ecological impacts when making late-stage design decisions, a monetary-based rating system is used to present environmental impacts. Environmental Priority Strategies in product design (EPS) is one such metric for ecological sustainability, and it contains an extensive database that links materials and processes with environmental impacts, quantified in terms of the Environmental Load Unit (ELU) [21]. In this rating system, one ELU is equivalent to an environmental damage cost of one Euro $(€)$.

Environmental impacts for a component such as the D-pillar of a vehicle come in the production phase, the use phase, and the end-of-life. EPS provides production impacts for creating various materials and manufacturing with different processes, as well as end-of-life impacts for different scenarios, including disposal in a landfill, combustion (for combustible materials), and reuse. The reuse scenario, which in- 
volves disassembling and recycling the components, is typically assigned negative ELUs, since reusing a material reduces future needs to produce usable materials from raw minerals. This study reports results for the landfill and reuse scenarios, demonstrating the benefits of designing with disassembly and reuse in mind.

The use phase of an automotive part is almost entirely dependent on mass, as higher-mass parts will require more fuel combustion over the life of the vehicle. Studies consistently indicate that a $10 \%$ mass reduction on a vehicle results in a $7 \%$ reduction in fuel consumption [23]. This is extrapolated and scaled for every percentage of mass change in this study. Using the assumptions that the full vehicle mass is 1800 kilograms, the baseline fuel consumption is 10 liters per 100 kilometers, and the baseline calculation that the D-pillar weighs 4.78 kilograms, the impact of the D-pillar on fuel consumption is calculated. The use phase impact is then calculated using the assumption that an average vehicle drives 300,000 kilometers over its lifetime and the EPS specification that unleaded petroleum costs the environment 1.12 ELU per kilogram. The environmental impact of the use phase is thus calculated by the amount of fuel required by the weight of the D-pillar over the life of the vehicle, compared to how much fuel would be consumed if the vehicle had no right-side D-pillar.

\subsection{Optimization Framework}

A multi-objective optimization formulation has been developed around the use of these three modeling tools. To simplify the optimization problem, the response surface method (RSM) is used on the computationally-intensive model of variation propagation in RD\&T, where each simulation uses the Monte Carlo method with 5000 points. Based on 3600 complete simulations with full-factorial sampling of the two input tolerances on a range of 0.05 to 3.00 millimeters, a polynomial response surface was fit using linear regression to generate a closed-form mathematical function for the probability of unacceptable critical dimensions in the part as a function of the input tolerances. This surrogate model is given as Equation (2), where $\varphi$ is the percentage of unacceptable parts and $t_{1}$ and $t_{2}$ are the input tolerances for the mating surfaces and support points, respectively. The model fits the data with a 0.9985 coefficient of determination, suggesting that the structure of the model is acceptable.

$$
\varphi=\left(0.71 t_{1}-7.96 t_{2}+29.51 t_{2}^{2}-6.32 t_{2}^{3}-0.96\right) / 100
$$

Using this surrogate model, a cost function is formulated with respect to tolerances and the percentage of discarded parts, shown in Equation (3) where $C$ is the economic cost in Euros and $C_{m a t}$ is the cost of the materials in Euros, taken from [24]. The final term on the right side of the equation $(1+\varphi)$ represents a multiplier to account for discarded products, as a larger percentage of discarded products raises the effective cost of producing acceptable products.

$$
C=\left(C_{m a t}+1 / t_{1}+1 / t_{2}\right)(1+\varphi)
$$

An additional model for ecological impact is formulated using the percentage of discarded parts and each of the factors from EPS. This is given as Equation (4), where 
$E$ is ecological impact in ELUs and is a function of mass $m$ and ecological factors $E_{i}$ relating to material production, manufacturing process, the use phase, and end-of-life.

$$
E=m\left(E_{\text {mat }}+E_{\text {proc }}+E_{\text {eol }}\right)(1+\varphi)+E_{\text {use }}
$$

Combining these models, a bi-objective optimization problem can be solved, formulated as Equation (5).

$$
\min _{t_{1}, t_{2}} w_{c} C+w_{e} E
$$

Here, the two components of the objective function are economic cost $C$ measured in Euros, and ecological impact $E$ measured in ELUs, each with its associated weighting parameter $w$. Optimization is performed with respect to the tolerance input variables $t_{1}$ and $t_{2}$.

\section{$4 \quad$ Results}

Multi-objective optimization results are commonly presented as Pareto frontiers, where each point on a curve represents a Pareto-optimal design that cannot be improved for one objective without a sacrifice to the other. Results for the D-pillar case study are presented in this section, starting with the baseline scenario and extending with a parametric study on material choice.

\subsection{D-pillar Design Costs and Ecological Impact}

The baseline D-pillar is constructed with mild steel sheet metal and an allowance of one millimeter of variance in all three critical dimensions. The Pareto frontier demonstrating the tradeoff between cost and environmental impact is presented in Figure 3.

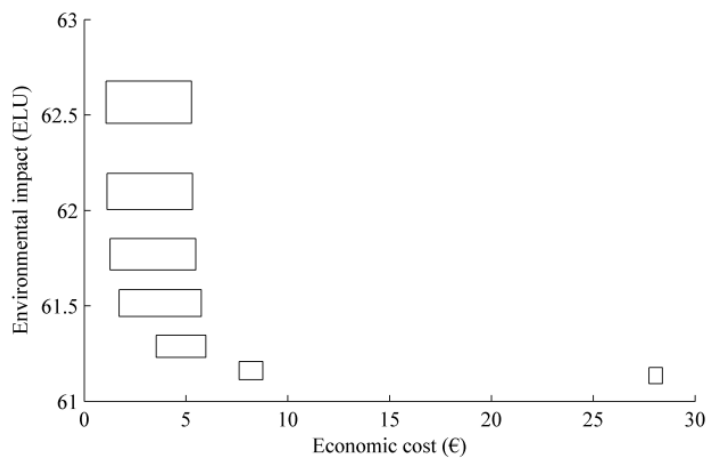

Fig. 3. Pareto frontier for baseline D-pillar, end-of-life landfill disposal

Each Pareto-optimal solution is represented in Figure 3 by a box, the dimensions of which indicate the optimizing tolerances. The horizontal width of the box represents $t_{1}$ (a wider box indicates a wider tolerance), and the vertical height represents $t_{2}$. Here, the upper-left solution corresponds with a purely cost-minimizing objective, and the 
lower-right solution minimizes environmental impact. The results shown here correspond with end-of-life disposal in a landfill; when reusing the materials is possible, the optimal economic costs stay the same while the environmental impacts are lowered by $8 \%$. Combustion of mild steel is not feasible.

\subsection{D-pillar Design with Parametric Variation of Material Choice}

The choice of material in this case study is important to the optimization solutions, as it affects outcomes such as part mass, and therefore the use phase ecological impact, as well as ecological sustainability impacts of extraction, production, and end-of-life disposal. Further, the material thickness is adjusted for each material based on the yield strength to ensure that the part can withstand the same compressive forces as a steel component (e.g., for a rollover/roof-crush test). Data on the strength, density, cost, and ecological impacts of five common automotive body materials were found in [24] and [21], and the resulting Pareto frontiers from optimizing the D-pillar using these materials are given in Figure 4, assuming end-of-life disposal in a landfill.

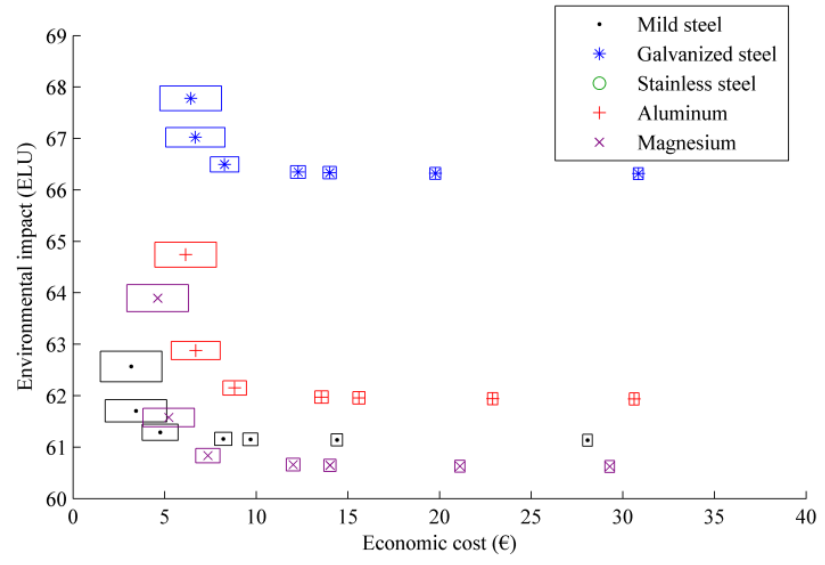

Fig. 4. Pareto frontiers of D-pillar varying material

Stainless steel has a much higher ecological impact of production than the other materials, and so the entire curve is off the visible chart in an environmental impact range of 340 to 380 ELUs. Like in Figure 3, Figure 4 presents data points as boxes where the widths and heights represent the corresponding optimal $t_{1}$ and $t_{2}$ values. Changing the end-of-life behavior from landfill disposal to reuse reduces the ecological impact by $14 \%, 63 \%, 27 \%$, and $29 \%$ for the latter four materials, respectively.

\section{Discussion}

The convexity of the bi-objective optimization results for the D-pillar tolerances demonstrates a clear tradeoff between economic costs to the manufacturer and ecological impact to society. The lower-right corner of Figure 3 shows that at high manufacturing costs, the tightest tolerances are associated with the lowest environmental im- 
pact. As the weighting of the objective function shifts towards economic costs, $t_{1}$ is observed to increase, followed by an increase in $t_{2}$. Even when the environmental impact does not affect the objective function, $t_{2}$ never reaches its maximum allowed value, shown by the box in the upper-left corner of the plot. This is attributed to the economic cost of discarding a large number of faulty parts when $t_{2}$ is high. Designing parts in which the materials can be reused rather than discarded is also beneficial, as it reduces the environmental impact by up to $63 \%$.

Parametrically varying the material choice shows that, for cost-minimizing firms, mild steel is the best option for the D-pillar. In cases where the environmental impact is more important to the manufacturer and costs are more flexible, magnesium becomes a better choice with tighter tolerances. In a scenario where the use phase becomes more important, e.g., if the number of kilometers driven increases substantially from the assumption of 300,000, lighter materials such as aluminum and magnesium may become more attractive than the mild steel. Likewise, in cases where corrosion of the part is a concern, galvanized or stainless steels may become better choices. When the design allows for reuse of the material, magnesium becomes the best choice.

\section{Conclusions}

While the specific results presented in this paper rely on assumptions built into the models, the framework provides new and important insights into how late-stage design choices should be considered with respect to internal manufacturing costs and external ecological costs. The automotive panel case shows a substantial tradeoff between economic and ecological costs resulting from tolerance and material choices, and further research is planned to reveal the impacts of additional sustainability decisions by designers and policymakers. As legislation and rising consumer interests in ecological sustainability continue to affect the market, this design approach will become more common for firms seeking to maximize profits in a competitive market.

Acknowledgments. The authors acknowledge valuable intellectual and modeling contributions from Kristina Wärmefjord and Lars Lindkvist of the Department of Product and Production Development at Chalmers. This work, carried out at the Wingquist Laboratory VINN Excellence Centre within the Area of Advance - Production at the Chalmers University of Technology, in Gothenburg, Sweden, was supported by the Swedish Governmental Agency for Innovation Systems (VINNOVA). That support is gratefully acknowledged.

\section{References}

1. Hong, Y.S., Chang, T.C.: A Comprehensive Review of Tolerancing Research. Int. J. Prod. Res. 40, 2425-2459 (2002)

2. Fortini, E.T.: Dimensioning for Interchangeable Manufacture. Industrial Press, New York (1967)

3. Bjørke, O.: Computer-Aided Tolerancing. ASME Press, New York (1989) 
4. Turner, J.U., Wozny, M.J.: Tolerances in Computer-Aided Geometric Design. The Visual Computer, 3, 214-226 (1987)

5. Shah, J.J., Ameta, G., Shen, Z., Davidson, J.: Navigating the Tolerance Analysis Maze. CAD and App. 4, 705-718 (2007)

6. Söderberg, R., Lindkvist, L.: Computer Aided Assembly Robustness Evaluation. J. Eng. Des. 10, 165-181 (1999)

7. Chase, K.W., Parkinson, A.R.: A Survey of Research in the Application of Tolerance Analysis to the Design of Mechanical Assemblies. Res. in Eng. Des. 3, 23-37 (1991)

8. Lööf, J., Hermansson, T., Söderberg, R.: An Efficient Solution to the Discrete Least-Cost Tolerance Allocation Problem with General Loss Functions. In: Davidson, J.K. (ed.) Models for Computer Aided Tolerancing in Design and Manufacturing, pp. 1148-1158. Springer, Dordrecht (2007)

9. Ostwalt, P.F., Huang, J.: A Method for Optimal Tolerance Selection. J. Eng. for Industry. 99, 558-565 (1977)

10. Choi, H.G.R., Park, M.H., Salisbury, E.: Optimal Tolerance Allocation with Loss Functions. J. Man. Sci. Eng. 122, 529-535 (2000)

11. Ding, Y., Jin, J., Ceglarek, D., Shi, J.: Process-Oriented Tolerance Synthesis for Multistage Manufacturing Systems. In: ASME International Mechanical Engineering Congress and Exposition (2000)

12. Li, Z., Izquierdo, L.E., Kokkolaras, M., Hu, S.J., Papalambros, P.Y.: Multiobjective Optimization for Integrated Tolerance Allocation and Fixture Layout Design in Multistation Assembly. J. Man. Sci. Eng. 130, 0445011-0445016 (2008)

13. Soderberg, R.: Tolerance Allocation Considering Customer and Manufacturer Objectives. In: Gilmore, B.J. Advances in Design Automation, DE-vol. 65-2, pp. 149-157. ASME, Albuquerque (1993)

14. Cheng, B.W., Maghsoodloo, S.: Optimization of Mechanical Assembly Tolerances by Incorporating Taguchi's Quality Loss Function. J. Man. Sys. 14, 264-276 (1995)

15. Jeang, A.: An Approach of Tolerance Design for Quality Improvement and Cost Reduction. Int. J. of Prod. Res. 35, 1193-1211 (1997)

16. Taguchi, G., Elsayed, E.A., Hsiang, T.: Quality Engineering in Production Systems. McGraw-Hill, Columbus (1989)

17. Evans, D.H.: Statistical Tolerancing: The State of the Art, Part I: Background. J. Qual. Tech. 6, 188-195 (1974)

18. Kane, V.E.: Process Capability Indices. J. Qual. Tech. 18, 41-52 (1986)

19. Vigon, B.W., Tolle, D.A., Cornaby, B.W., Latham, H.C.: Life-Cycle Assessment: Inventory Guidelines and Principles. Technical Report, Environmental Protection Agency, Cincinnati (1993)

20. Taghizadeh, A., Dagman, A., Almefelt, L.: Evaluation of Four Tools for Environmental Impact Life Cycle Assessment in Sustainable Product Development. In: 17th CIRP International Conference on Life Cycle Engineering, University of Technology press (2010)

21. Steen, B.: A Systematic Approach to Environmental Priority Strategies in Product Development (EPS). Version 2000 - General System Characteristics. Technical report, Chalmers University of Technology, Technical Environmental Planning (1999)

22. The Netherlands Ministry of Housing, Spatial Planning and the Environment: Ecoindicator 99 Manual for Designers. Manual (2000)

23. Lutsey, N.: Review of Technical Literature and Trends Related to Automobile MassReduction Technology. Technical report, California Air Resources Board (2010)

24. Ullman, D.G.: The Mechanical Design Process, Third Edition. McGraw Hill, Boston (2003) 